\title{
Proper holomorphic mappings between symmetrized ellipsoids
}

\author{
PAWEe ZAPAŁOWSKI
}

\begin{abstract}
We characterize the existence of proper holomorphic mappings in the special class of bounded $(1,2, \ldots, n)$-balanced domains in $\mathbb{C}^{n}$, called the symmetrized ellipsoids. Using this result we conclude that there are no non-trivial proper holomorphic self-mappings in the class of symmetrized ellipsoids. We also describe the automorphism groups of these domains.
\end{abstract}

Mathematics Subject Classification (2010). 32H35, 32A07.

Keywords. Proper holomorphic mappings, Symmetrized ellipsoids, Quasi balanced domains, Group of automorphisms.

1. Introduction and statement of results. For $n \geqslant 2$ and $p>0$ let

$$
\mathbb{B}_{p, n}:=\left\{\left(z_{1}, \ldots, z_{n}\right) \in \mathbb{C}^{n}: \sum_{j=1}^{n}\left|z_{j}\right|^{2 p}<1\right\}
$$

denote the generalized complex ellipsoid. We shall write $\mathbb{B}_{n}:=\mathbb{B}_{1, n}, \mathbb{T}:=\partial \mathbb{B}_{1}$. Note that $\mathbb{B}_{p, n}$ is a bounded, complete Reinhardt domain.

Let $\pi_{n}=\left(\pi_{n, 1}, \ldots, \pi_{n, n}\right): \mathbb{C}^{n} \rightarrow \mathbb{C}^{n}$ be defined as follows

$$
\pi_{n, k}(z)=\sum_{1 \leqslant j_{1}<\cdots<j_{k} \leqslant n} z_{j_{1}} \ldots z_{j_{k}}, \quad 1 \leqslant k \leqslant n, z=\left(z_{1}, \ldots, z_{n}\right) \in \mathbb{C}^{n} .
$$

Note that $\pi_{n}$ is a proper holomorphic mapping with multiplicity $n !,\left.\pi_{n}\right|_{\mathbb{B}_{p, n}}$ : $\mathbb{B}_{p, n} \rightarrow \pi_{n}\left(\mathbb{B}_{p, n}\right)$ is proper too.

The set

$$
\mathbb{E}_{p, n}:=\pi_{n}\left(\mathbb{B}_{p, n}\right)
$$

is called the symmetrized $(p, n)$-ellipsoid. Note that $\mathbb{E}_{p, n}$ is a bounded $(1,2, \ldots$, $n$ )-balanced domain (recall that a domain $D \subset \mathbb{C}^{n}$ is called the $\left(k_{1}, \ldots, k_{n}\right)$ -

The research was partially supported by the Research Grant No. N N201 361436 of the Polish Ministry of Science and Higher Education. 
balanced, where $k_{1}, \ldots, k_{n} \in \mathbb{N}$, if $\left(\lambda^{k_{1}} z_{1}, \ldots, \lambda^{k_{n}} z_{n}\right) \in D$ for any $\left(z_{1}, \ldots, z_{n}\right) \in$ $D$ and $\left.\lambda \in \overline{\mathbb{B}}_{1}\right)$. Geometric properties of $\mathbb{E}_{p, n}$ were studied in [12]. Here we answer some of the open questions posed there. As the definition of the symmetrized ellipsoid is similar to the one of the symmetrized polydisc $\mathbb{G}_{n}:=\pi_{n}\left(\mathbb{B}_{1}^{n}\right)$, which has drawn a lot of attention recently (see $[1,4,6,10]$ and the references given there), it is quite natural to ask which properties of the symmetrized polydisc are inherited by the symmetrized ellipsoids.

Our aim is to give necessary and sufficient condition for existence of the proper holomorphic mappings between the symmetrized ellipsoids.

Here is some notation. Let $\mathfrak{S}_{n}$ denote the group of permutations of the set $\{1, \ldots, n\}$. For $\sigma \in \mathfrak{S}_{n}, z=\left(z_{1}, \ldots, z_{n}\right) \in \mathbb{C}^{n}$ denote $z_{\sigma}:=\left(z_{\sigma(1)}, \ldots, z_{\sigma(n)}\right)$. Next, for any $A \subset \mathbb{C}$ put $A_{*}:=A \backslash\{0\}, A_{*}^{n}:=\left(A_{*}\right)^{n}$. Moreover, for any $z=\left(z_{1}, \ldots, z_{n}\right) \in \mathbb{C}^{n}, w=\left(w_{1}, \ldots, w_{n}\right) \in \mathbb{C}^{n}, t \in \mathbb{C}$ and $r>0$ we put $z w:=\left(z_{1} w_{1}, \ldots, z_{n} w_{n}\right), t z:=\left(t z_{1}, \ldots, t z_{n}\right)$, and $z^{r}:=\left(z_{1}^{r}, \ldots, z_{n}^{r}\right)$.

Remark 1. (a) Let $l \in \mathbb{N}$. Observe that $\mathbb{C}^{n} \ni z \mapsto \pi_{n}\left(z^{l}\right) \in \mathbb{C}^{n}$ is a symmetric polynomial mapping. According to the fundamental theorem of symmetric polynomials (see, e.g., [9]) there is a unique polynomial mapping $P_{l}: \mathbb{C}^{n} \rightarrow \mathbb{C}^{n}$ such that $\pi_{n}\left(z^{l}\right)=P_{l}\left(\pi_{n}(z)\right), z \in \mathbb{C}^{n}$. In particular, $P_{l}\left(\mathbb{E}_{p, n}\right)=\mathbb{E}_{p / l, n}$ for any $p>0$.

(b) Fix $A, B, C \in \mathbb{C}$ and put $L:=\left(L_{1}, \ldots, L_{n}\right): \mathbb{C}^{n} \rightarrow \mathbb{C}^{n}$, where

$$
L_{j}(z):=A \sum_{k=1}^{n} z_{k}+B z_{j}+C, \quad z=\left(z_{1}, \ldots, z_{n}\right) \in \mathbb{C}^{n}, \quad j=1, \ldots, n .
$$

Observe that $\pi_{n} \circ L$ is a symmetric polynomial mapping. According to the fundamental theorem of symmetric polynomials there is a unique polynomial mapping $S_{L}: \mathbb{C}^{n} \rightarrow \mathbb{C}^{n}$ such that $\pi_{n} \circ L=S_{L} \circ \pi_{n}$.

Now we are in position to formulate our main result.

Theorem 2. There exists proper holomorphic mapping $f: \mathbb{E}_{p, n} \rightarrow \mathbb{E}_{q, n}$ iff $p / q \in \mathbb{N}$. Furthermore, if $p / q \in \mathbb{N}$, the only proper holomorphic mappings $f: \mathbb{E}_{p, n} \rightarrow \mathbb{E}_{q, n}$

(a) in case $p \neq 1$, or $q \neq 1 /(2 m), m \in \mathbb{N}$, or $n \neq 2$ are of the form

$$
f=P_{p / q} \circ \phi \text {, }
$$

where $P_{p / q}$ is as in Remark $1(a)$ and $\phi$ is an automorphism of $\mathbb{E}_{p, n}$;

(b) in case $p=1, q=1 /(2 m), m \in \mathbb{N}$, and $n=2$ are of the form (1) or

$$
f=P_{m} \circ \phi_{I I I} \circ P_{2} \circ \phi_{I I},
$$

where $\phi_{I I}\left(\right.$ resp. $\left.\phi_{I I I}\right)$ is the automorphism of $\mathbb{E}_{1,2}$ (resp. $\mathbb{E}_{1 / 2,2}$ ) defined in Corollary 4.

Similar classification for the class of generalized complex ellipsoids (with not necessarily equal exponents on each coordinate) was done in [8] (the case of positive integer exponents) and [5] (case of positive real exponents).

An immediate consequence of Theorem 2 is the following Alexander-type theorem for the symmetrized ellipsoids saying that every proper holomorphic self-map of the symmetrized ellipsoid is an automorphism. 
Corollary 3. Let $f: \mathbb{E}_{p, n} \rightarrow \mathbb{E}_{p, n}$ be a proper holomorphic self-mapping. Then $f$ is an automorphism.

A theorem of that type was obtained in the case of $\mathbb{B}_{n}$ in [3] and its generalization on complex ellipsoids was done in [8] and [5]. Recently, a similar result was obtained in [7] for the tetrablock, which is a $(1,1,2)$-balanced domain in $\mathbb{C}^{3}$. Characterization of proper holomorphic self-mappings of symmetrized polydisc is done in [6].

Furthermore, from the proof of Theorem 2, the automorphisms group of $\mathbb{E}_{p, n}$ may be easily derived.

Corollary 4. (a) If $p \neq 1$ and $(p, n) \neq(1 / 2,2)$ then the only automorphisms of $\mathbb{E}_{p, n}$ are of the form

$$
\phi_{I}\left(z_{1}, z_{2}, \ldots, z_{n}\right)=\left(\zeta z_{1}, \zeta^{2} z_{2}, \ldots, \zeta^{n} z_{n}\right), \quad\left(z_{1}, z_{2} \ldots, z_{n}\right) \in \mathbb{E}_{p, n},
$$

where $\zeta \in \mathbb{T}$.

(b) The only automorphisms of $\mathbb{E}_{1, n}$, are of the form

$$
\begin{aligned}
\phi_{I I}(z)= & \left(\frac{S_{L_{\varphi_{I I}}, 1}(z)}{n\left(1-a_{0} z_{1}\right)}, \ldots, \frac{S_{L_{\varphi_{I I}}, n}(z)}{n^{n}\left(1-a_{0} z_{1}\right)^{n}}\right), \\
& z=\left(z_{1}, \ldots, z_{n}\right) \in \mathbb{E}_{1, n},
\end{aligned}
$$

where $S_{L_{\varphi_{I I}}}=\left(S_{L_{\varphi_{I I}}, 1}, \ldots, S_{L_{\varphi_{I I}}, n}\right)$ is the polynomial mapping as in Remark $1(b)$ induced by $L_{\varphi_{I I}}=\left(L_{\varphi_{I I}, 1}, \ldots, L_{\varphi_{I I}, n}\right): \mathbb{C}^{n} \rightarrow \mathbb{C}^{n}$, where

$L_{\varphi_{I I}, j}\left(z_{1}, \ldots, z_{n}\right):=\zeta_{1}\left(\sum_{k=1}^{n} z_{k}-n a_{0}\right)+\zeta_{2} \sqrt{1-n a_{0}^{2}}\left(\sum_{k=1}^{n} z_{k}-n z_{j}\right)$,

for some $\zeta_{1}, \zeta_{2} \in \mathbb{T}, a_{0} \in \mathbb{R}, a_{0}^{2}<\frac{1}{n}$.

(c) The only automorphisms of $\mathbb{E}_{1 / 2,2}$ are of the form (2) or

$$
\phi_{I I I}\left(z_{1}, z_{2}\right)=\left(\zeta z_{1}, \zeta^{2}\left(\frac{1}{4} z_{1}^{2}-z_{2}\right)\right), \quad\left(z_{1}, z_{2}\right) \in \mathbb{E}_{1 / 2,2},
$$

where $\zeta \in \mathbb{T}$.

Remark 5. It should be mentioned that the automorphisms of the form (2) are special cases of the automorphisms of the form (3).

\section{Proofs.}

Remark 6. For a $\left(k_{1}, \ldots, k_{n}\right)$-balanced domain $D \subset \mathbb{C}^{n}$ one may define the generalized Minkowski functional

$\mu_{D}\left(z_{1}, \ldots, z_{n}\right):=\inf \left\{\lambda>0:\left(\lambda^{-k_{1}} z_{1}, \ldots, \lambda^{-k_{n}} z_{n}\right) \in D\right\}, \quad\left(z_{1}, \ldots, z_{n}\right) \in \mathbb{C}^{n}$.

Observe that for a $(1,2, \ldots, n)$-balanced domain $\mathbb{E}_{p, n}$ we have

$$
\mu_{\mathbb{E}_{p, n}}(z)=\max \left\{\left(\sum_{j=1}^{n}\left|w_{j}\right|^{2 p}\right)^{1 / 2 p}:\left(w_{1}, \ldots, w_{n}\right) \in \pi_{n}^{-1}(z)\right\}, \quad z \in \mathbb{C}^{n} .
$$

In particular, $\mu_{\mathbb{E}_{p, n}}$ is continuous. 
Proof of Theorem 2. If $p / q \in \mathbb{N}$ then $f(z):=P_{p / q}(z)$ is a proper holomorphic mapping between $\mathbb{E}_{p, n}$ and $\mathbb{E}_{q, n}$.

Assume $f: \mathbb{E}_{p, n} \rightarrow \mathbb{E}_{q, n}$ is proper and holomorphic. Since $\mu_{\mathbb{E}_{p, n}}$ is continuous, it follows from [7] that $f$ extends holomorphically past the boundary, $\partial \mathbb{E}_{p, n}$, of $\mathbb{E}_{p, n}$. Hence there is a domain $V \subset \mathbb{C}^{n}$ such that

- $V \cap \partial \mathbb{E}_{p, n} \neq \varnothing$,

- the mapping $\left.f\right|_{V}: V \rightarrow f(V)$ is biholomorphic,

- the mappings $\left.\pi_{n}\right|_{\pi_{n}^{-1}(V)}: \pi_{n}^{-1}(V) \rightarrow V$ and $\left.\pi_{n}\right|_{\pi_{n}^{-1}(f(V))}: \pi_{n}^{-1}(f(V)) \rightarrow$ $f(V)$ are biholomorphic.

Since $\pi_{n}\left(\partial \mathbb{B}_{p, n}\right)=\partial \mathbb{E}_{p, n}$, it is not restrictive to assume that for a domain $U:=\pi_{n}^{-1}(V) \subset \mathbb{C}^{n}$

- $U \cap \partial \mathbb{B}_{p, n} \neq \varnothing$,

- the mapping $U \ni z \stackrel{g_{p}}{\longmapsto} z^{p} \in g_{p}(U)$ is well defined and biholomorphic,

- the mapping $\pi_{n}^{-1}\left(f\left(\pi_{n}(U)\right)\right) \ni z \stackrel{g_{q}}{\longmapsto} z^{q} \in g_{q}\left(\pi_{n}^{-1}\left(f\left(\pi_{n}(U)\right)\right)\right)$ is well defined and biholomorphic.

Hence the mapping $\psi:=\left.\pi_{n}^{-1} \circ f \circ \pi_{n}\right|_{U}: U \rightarrow \psi(U)$ is well defined and biholomorphic. Consequently, the mapping $\varphi:=\left.g_{q} \circ \psi \circ g_{p}^{-1}\right|_{g_{p}(U)}$ is holomorphic and $\left.\varphi\right|_{g_{p}(U) \cap \mathbb{B}_{n}}$ is biholomorphic. As $\varphi\left(g_{p}(U) \cap \partial \mathbb{B}_{n}\right) \subset \partial \mathbb{B}_{n}$, it follows from [2] that $\varphi$ extends to an automorphism of $\mathbb{B}_{n}$, still denoted by $\varphi=\left(\varphi_{1}, \ldots, \varphi_{n}\right)$. Hence

$$
\pi_{n}\left(\varphi^{1 / q}(z)\right)=f\left(\pi_{n}\left(z^{1 / p}\right)\right), \quad z \in g_{p}(U) .
$$

We use the following lemma which will be proved afterwards.

Lemma 7. Let $\varphi$ be an automorphism of $\mathbb{B}_{n}$ which satisfies (5) and let $m:=1 / q, l:=1 / p$.

(a) If $m \notin \mathbb{N}$ then $m / l \in \mathbb{N}$ and, up to permutation of variables, $\varphi$ is of the form

$$
\varphi_{I}\left(z_{1}, \ldots, z_{n}\right)=\zeta\left(\eta_{1} z_{1}, \ldots, \eta_{n} z_{n}\right), \quad\left(z_{1}, \ldots, z_{n}\right) \in \mathbb{B}_{n}
$$

for some $\zeta, \eta_{j} \in \mathbb{T}, \eta_{j}^{m}=1, j=1, \ldots, n$.

(b) If $m \in \mathbb{N}$ then $l \in \mathbb{N}$ and $m / l \in \mathbb{N}$. Moreover,

(i) if $l=1$ and $n \geqslant 3$ then, up to permutation of variables and components, $\varphi$ is of the form $\varphi_{I I}=\left(\varphi_{I I, 1}, \ldots, \varphi_{I I, n}\right)$, where

$$
\begin{aligned}
\varphi_{I I, j}\left(z_{1}, \ldots, z_{n}\right)= & \frac{\eta_{j}}{n\left(1-a_{0} \sum_{k=1}^{n} z_{k}\right)}\left(\zeta_{1}\left(\sum_{k=1}^{n} z_{k}-n a_{0}\right)\right. \\
& \left.+\zeta_{2} \sqrt{1-n a_{0}^{2}}\left(\sum_{k=1}^{n} z_{k}-n z_{j}\right)\right), \\
& \left(z_{1}, \ldots, z_{n}\right) \in \mathbb{B}_{n},
\end{aligned}
$$

for some $a_{0} \in \mathbb{R}, a_{0}^{2}<\frac{1}{n}, \zeta_{1}, \zeta_{2}, \eta_{j} \in \mathbb{T}, \eta_{j}^{m}=1, j=1, \ldots, n$;

(ii) if $l \geqslant 2$ and $n \geqslant 3$ then, up to permutation of variables and components, $\varphi$ is of the form (6); 
(iii) if $l=1$ and $n=2$ then, up to permutation of variables and components, $\varphi$ is of the form (7); moreover, if $m$ is even then, additionally, up to permutation of variables and components, $\varphi$ is of the form

$$
\begin{aligned}
\varphi_{I I I}\left(z_{1}, z_{2}\right)= & \frac{1}{\sqrt{2}\left(1-a_{0}\left(z_{1}+z_{2}\right)\right)} \\
& \left(\zeta_{1}\left(z_{1}+z_{2}-2 a_{0}\right), \zeta_{2} \sqrt{1-2 a_{0}^{2}}\left(z_{1}-z_{2}\right)\right), \\
& \left(z_{1}, z_{2}\right) \in \mathbb{B}_{2},
\end{aligned}
$$

for some $\zeta_{1}, \zeta_{2} \in \mathbb{T}$ and $a_{0} \in \mathbb{R}, a_{0}^{2}<\frac{1}{2}$;

(iv) if $l=2$ and $n=2$ then, up to permutation of variables and components, $\varphi$ is of the form (6) or

$$
\varphi_{I V}\left(z_{1}, z_{2}\right)=\frac{\zeta}{\sqrt{2}}\left(z_{1}+z_{2}, \eta\left(z_{1}-z_{2}\right)\right), \quad\left(z_{1}, z_{2}\right) \in \mathbb{B}_{2},
$$

for some $\zeta, \eta \in \mathbb{T}, \eta^{m}=1$.

Remark 8. It should be mentioned that the automorphisms of the form (6) are special cases of the automorphisms of the form (7).

Note that for any automorphism $\varphi$ of $\mathbb{B}_{n}$ which satisfies (6), (7), or (9) respectively, there is an automorphism $\tilde{\varphi}$ of $\mathbb{B}_{n}$ such that $\pi_{n}\left(\varphi^{m}(z)\right)=$ $\pi_{n}\left(\tilde{\varphi}^{m}(z)\right)$ and $\pi_{n}\left(\tilde{\varphi}^{l}(z)\right)=\pi_{n}\left(\tilde{\varphi}^{l}\left(z_{\sigma}\right)\right)$ for any $z \in \mathbb{B}_{n}$, and $\sigma \in \mathfrak{S}_{n}$.

Indeed,

- in case of (6) it suffices to take $\tilde{\varphi}$ of the form (6) with $\eta_{j}=1, j=1, \ldots, n$. Then the relation $\phi \circ \pi_{n}=\pi_{n} \circ \tilde{\varphi}$ defines the automorphism $\phi_{I}$ of $\mathbb{E}_{p, n}$ of the form (2), which obviously satisfies the relation

$$
\phi\left(\pi_{n}\left(z^{1 / p}\right)\right)=\pi_{n}\left(\tilde{\varphi}^{1 / p}(z)\right), \quad z \in \mathbb{B}_{n} .
$$

- In case of (7) it suffices to take $\tilde{\varphi}$ of the form (7) with $\eta_{j}=1, j=1, \ldots, n$. Then the relation $\phi \circ \pi_{n}=\pi_{n} \circ \tilde{\varphi}$ defines the automorphism $\phi_{I I}$ of $\mathbb{E}_{1, n}$ of the form (3), which obviously satisfies the relation (10).

- In case of (9) it suffices to take $\tilde{\varphi}$ of the form (9) with $\eta^{2}=1$. Then the relation (10), which in this case has form

$$
\phi\left(\pi_{2}\left(z^{2}\right)\right)=\pi_{2}\left(\tilde{\varphi}^{2}(z)\right), \quad z \in \mathbb{B}_{2},
$$

defines the automorphism $\phi_{I I I}$ of $\mathbb{E}_{1 / 2,2}$ of the form (4).

It follows from Lemma 7 that $p / q=m / l \in \mathbb{N}$. Consequently, using (10),

$$
\begin{aligned}
f\left(\pi_{n}\left(z^{1 / p}\right)\right) & =\pi_{n}\left(\varphi^{1 / q}(z)\right)=\pi_{n}\left(\tilde{\varphi}^{1 / q}(z)\right)=\pi_{n}\left(\left(\tilde{\varphi}^{1 / p}(z)\right)^{p / q}\right) \\
& =P_{p / q}\left(\pi_{n}\left(\tilde{\varphi}^{1 / p}(z)\right)\right)=P_{p / q}\left(\phi\left(\pi_{n}\left(z^{1 / p}\right)\right)\right), \quad z \in g_{p}(U) .
\end{aligned}
$$

The identity principle implies that $f=P_{p / q} \circ \phi$ which ends the proof in the case, when equality (5) is satisfied by the automorphisms of the form (6), (7), or $(9)$.

In the case when equality (5) is satisfied by the automorphism of the form (8), the situation is slightly different and we proceed as follows. First observe 
that $\varphi_{I I I}=\varphi_{I V} \circ \varphi_{I I}$, where $\varphi_{I V}$ and $\varphi_{I I}$ are taken with $\eta=\eta_{1}=\eta_{2}=1$. Since $m$ is even, $m=2 m^{\prime}$ for some $m^{\prime} \in \mathbb{N}$. Then the previous cases imply

$$
\begin{aligned}
f\left(\pi_{2}(z)\right) & =\pi_{2}\left(\varphi_{I I I}^{2 m^{\prime}}(z)\right)=\pi_{2}\left(\varphi_{I V}^{2 m^{\prime}}\left(\varphi_{I I}(z)\right)\right)=P_{m^{\prime}}\left(\pi_{2}\left(\varphi_{I V}^{2}\left(\varphi_{I I}(z)\right)\right)\right) \\
& =P_{m^{\prime}}\left(\phi_{I I I}\left(\pi_{2}\left(\varphi_{I I}^{2}(z)\right)\right)\right)=P_{m^{\prime}}\left(\phi_{I I I}\left(P_{2}\left(\phi_{I I}\left(\pi_{2}(z)\right)\right)\right), \quad z \in g_{1}(U),\right.
\end{aligned}
$$

whence $f=P_{m^{\prime}} \circ \phi_{I I I} \circ P_{2} \circ \phi_{I I}$.

Remark 9. Following [11] any automorphism $\varphi=\left(\varphi_{1}, \ldots, \varphi_{n}\right)$ of the unit ball is of the form

$$
\varphi_{j}(z)=\frac{\sum_{k=1}^{n} q_{j, k}\left(z_{k}-a_{k}\right)}{R\left(1-\sum_{k=1}^{n} \bar{a}_{k} z_{k}\right)}, \quad z=\left(z_{1}, \ldots, z_{n}\right) \in \mathbb{B}_{n}, j=1, \ldots, n,
$$

where $a=\left(a_{1}, \ldots, a_{n}\right) \in \mathbb{B}_{n}$ is arbitrary, $Q=\left[q_{j, k}\right]$ and $R$ are respectively a $n \times n$ matrix and a constant such that

$$
\bar{Q}\left(\mathbb{I}_{n}-\bar{a}^{t} a\right)^{t} Q=\mathbb{I}_{n}, \quad \bar{R}\left(1-{ }^{t} a \bar{a}\right) R=1,
$$

where $\mathbb{I}_{n}$ is the unit $n \times n$ matrix, whereas $\bar{A}$ (resp. ${ }^{t} A$ ) is the conjugate (resp. transpose) of an arbitrary matrix $A$. Moreover, $a, Q$, and $R$ satisfy

$$
\left\{\begin{array}{l}
{ }^{t} Q \bar{Q}-|R|^{2} \bar{a}^{t} a=\mathbb{I}_{n} \\
|R|^{2}-{ }^{t} a{ }^{t} Q \bar{Q} \bar{a}=1 \\
{ }^{t} Q \bar{Q} \bar{a}=|R|^{2} a
\end{array} .\right.
$$

In particular, $Q$ is unitary if $a=0$.

Proof of Lemma \%. In the proof we will use the form of automorphism $\varphi$ of $\mathbb{B}_{n}$ as in Remark 9.

Ad (a). Assume $m \notin \mathbb{N}$. Note that the function on the right side of (5) is well defined on any domain $D \subset \mathbb{B}_{n} \cap \mathbb{C}_{*}^{n}$ such that the fiber $D_{j}:=\{\lambda \in$ $\left.\mathbb{C}:\left(z_{1}, \ldots, z_{j-1}, \lambda, z_{j+1}, \ldots, z_{n}\right) \in D\right\}$ is connected and simply connected for $j=1, \ldots, n$. In particular, the function $D \ni z \mapsto\left(\prod_{j=1}^{n} \varphi_{j}(z)\right)^{m}$ is holomorphic. Assumption $m \notin \mathbb{N}$ implies that

$$
\varphi\left(\mathbb{B}_{n} \cap \mathbb{C}_{*}^{n}\right) \subset \mathbb{C}_{*}^{n} .
$$

We show that $\varphi$ is of the form (6).

First we show that $a=0$ and for any $j \in\{1, \ldots, n\}$ there exists a unique $k$ such that $q_{j, k} \neq 0$.

Indeed, suppose the contrary. Then either

- there are $j, k_{1}, k_{2}$ with $k_{1} \neq k_{2}$ and $q_{j, k_{1}} \neq 0 \neq q_{j, k_{2}}$, or

- there are $j, k_{1}$ with $q_{j, k_{1}} \neq 0 \neq a_{k_{1}}$ ( since $\varphi$ is one-to-one mapping, for any $k$ there is a $j$ such that $\left.q_{j, k} \neq 0\right)$.

In both cases one may define

$$
w_{k_{1}}:=a_{k_{1}}-\sum_{k \neq k_{1}} \frac{q_{j, k}}{q_{j, k_{1}}}\left(w_{k}-a_{k}\right) \neq 0,
$$

provided $w_{k} \in \mathbb{C}_{*}, k \neq k_{1}$, are chosen close to $a_{k}$ enough. Clearly, one may assume that $w:=\left(w_{1}, \ldots, w_{n}\right) \in \mathbb{B}_{n}$. Consequently, $w \in \mathbb{B}_{n} \cap \mathbb{C}_{*}^{n}$ with $\varphi_{j}(w)=$ 0 - a contradiction with (12). 
First equality in (11) implies that $\varphi\left(z_{1}, \ldots, z_{n}\right)=\left(\zeta_{1} z_{\sigma(1)}, \ldots, \zeta_{n} z_{\sigma(n)}\right)$ for some $\sigma \in \mathfrak{S}_{n}$ and $\zeta_{j} \in \mathbb{T}, j=1, \ldots, n$. Moreover, (5) and the identity principle imply that for any $\omega \in \mathfrak{S}_{n}$ there is $\tau \in \mathfrak{S}_{n}$ such that

$$
\left(\zeta_{1}^{m} z_{\omega(1)}^{m}, \ldots, \zeta_{n}^{m} z_{\omega(n)}^{m}\right)=\left(\zeta_{\tau(1)}^{m} z_{\tau(1)}^{m}, \ldots, \zeta_{\tau(n)}^{m} z_{\tau(n)}^{m}\right), \quad z \in \mathbb{B}_{n},
$$

whence we conclude that $\zeta_{j}^{m}=\zeta_{k}^{m}=\tilde{\zeta}$ for $j, k=1, \ldots, n$, i.e., $\varphi$ is of the form (6).

Finally, observe that (5) implies that

$$
f\left(\pi_{n}\left(z^{l}\right)\right)=\pi_{n}\left(\tilde{\zeta} z^{m}\right), \quad z \in g_{p}(U) .
$$

Hence $f_{n}\left(z_{1}, \ldots, z_{n}\right)=\tilde{\zeta}^{n} z_{n}^{m / l}$. Since $f$ is holomorphic on $\mathbb{E}_{p, n}$, we conclude that $m / l \in \mathbb{N}$.

Ad (b). Assume now $m \in \mathbb{N}$. Then $\pi_{n} \circ \varphi^{m}: \mathbb{B}_{n} \rightarrow \mathbb{E}_{1 / m, n}$ is the proper holomorphic mapping with multiplicity $n ! m$. Thus equality (5) extends on $\mathbb{B}_{n}$ and implies that $g_{1 / p}: \mathbb{B}_{n} \rightarrow \mathbb{B}_{p, n}$ is the proper holomorphic mapping with multiplicity $1 / p=l \in \mathbb{N}, m=k l$, where $k \in \mathbb{N}$ is the multiplicity of $f$.

The equality (5) and the identity principle imply that for any $\sigma \in \mathfrak{S}_{n}$ and $\xi=\left(\xi_{1}, \ldots, \xi_{n}\right) \in \mathbb{T}^{n}, \xi_{j}^{l}=1, j=1, \ldots, n$, there are $\tau \in \mathfrak{S}_{n}$ and $\eta=$ $\left(\eta_{1}, \ldots, \eta_{n}\right) \in \mathbb{T}^{n}, \eta_{j}^{m}=1, j=1, \ldots, n$, such that

$$
\varphi(z)=\eta \varphi_{\tau}\left(\xi z_{\sigma}\right), \quad z \in \mathbb{B}_{n} .
$$

Observe that condition (13) implies that $a=\left(a_{0}, \ldots, a_{0}\right)$ for some $a_{0} \in \frac{1}{\sqrt{n}} \mathbb{B}_{1}$. Indeed, for any $\sigma \in \mathfrak{S}_{n}$ there are $\tau \in \mathfrak{S}_{n}$ and $\eta \in \mathbb{T}^{n}$ such that

$$
0=\varphi(a)=\eta \varphi_{\tau}\left(a_{\sigma}\right) .
$$

Hence $\varphi\left(a_{\sigma}\right)=0$, i.e., $a=a_{\sigma}$.

Moreover, for $l>1 \varphi$ is unitary. Indeed, suppose $a \neq 0$. Then there is $\xi \in \mathbb{T}^{n}, \xi^{l}=1$, with $\xi a \neq a$. Hence $0=\varphi(a)=\eta \varphi_{\tau}(\xi a)$-contradiction, since $\varphi_{\tau}(a)=0$.

Ad (i). The equality (5) implies that $z \mapsto \pi_{n}\left(\varphi^{m}(z)\right)$ is symmetric polynomial mapping. In particular, the polynomial

$$
\mathbb{C}^{n} \ni\left(z_{1}, \ldots, z_{n}\right) \mapsto \prod_{j=1}^{n}\left(\sum_{k=1}^{n} q_{j, k}\left(z_{k}-a_{0}\right)\right)^{m}
$$

is symmetric.

Let $N_{j}:=\#\left\{k: q_{j, k} \neq 0\right\}, j=1,2, \ldots, n$, and let

$$
N_{Q}:=\min \left\{\#\left\{z: \exists_{j, k} \eta_{j} q_{j, k}=z\right\}: \eta_{j} \in \mathbb{T}, \eta_{j}^{m}=1, j=1, \ldots, n\right\} .
$$

The matrix $\hat{Q}=\hat{Q}(\eta)=\left[\eta_{j} q_{j, k}\right]$ for $\eta=\left(\eta_{1}, \ldots, \eta_{n}\right) \in \mathbb{T}^{n}, \eta_{j}^{m}=1, j=$ $1, \ldots, n$, such that $N_{Q}=\#\left\{z: \exists_{j, k} \eta_{j} q_{j, k}=z\right\}$ we call the reduced matrix of the matrix $Q$.

Note that the polynomial (14) has at most $n$ different - up to multiplicative constant - affine factors, i.e., factors of the form $b_{1} z_{1}+\cdots+b_{n} z_{n}+b_{0}$. Consequently, the symmetry of the polynomial (14) implies that 
(i-i) $N_{1}=N_{2}=\cdots=N_{n}=n$, or

(i-ii) $N_{1}=N_{2}=\cdots=N_{n}=n-1$, or

(i-iii) $N_{1}=N_{2}=\cdots=N_{n}=1$.

We consider these cases separately.

Case (i-i). Let $N_{j}=n, j=1,2, \ldots, n$. We consider three subcases.

- $N_{Q}=1$. Then the first equality in (11) leads to a contradiction.

- $N_{Q}=2$ and one of the entries in the reduced matrix $\hat{Q}$ appears in some row exactly once. Because of the symmetry of the polynomial (14) we infer that it is the case in every row and in every column. Hence we may assume that

$$
q_{j, k}=\left\{\begin{array}{ll}
\eta_{j} \alpha, & \text { if } j \neq k \\
\eta_{j} \beta, & \text { if } j=k
\end{array},\right.
$$

for some $\alpha, \beta \in \mathbb{C}, \alpha \neq \beta$. Then equalities (11) give

$$
\left\{\begin{array}{l}
(n-1)|\alpha|^{2}+|\beta|^{2}=1+\left|a_{0}\right|^{2}|R|^{2} \\
(n-2)|\alpha|^{2}+2 \operatorname{Re}(\alpha \bar{\beta})=\left|a_{0}\right|^{2}|R|^{2} \\
|R|^{2}-n\left|a_{0}\right|^{2}|(n-1) \alpha+\beta|^{2}=1 \\
\bar{a}_{0}|(n-1) \alpha+\beta|^{2}=a_{0}|R|^{2}
\end{array}\right.
$$

which, after elementary calculation, implies

$$
\alpha=\frac{1}{n}\left(\frac{\zeta_{1}}{\sqrt{1-n a_{0}^{2}}}+\zeta_{2}\right), \quad \beta=\frac{1}{n}\left(\frac{\zeta_{1}}{\sqrt{1-n a_{0}^{2}}}-(n-1) \zeta_{2}\right)
$$

for some $a_{0} \in \mathbb{R}, a_{0}^{2}<\frac{1}{n}$, and $\zeta_{1}, \zeta_{2} \in \mathbb{T}$. Consequently,

$$
q_{j, k}= \begin{cases}\frac{\eta_{j}}{n}\left(\frac{\zeta_{1}}{\sqrt{1-n a_{0}^{2}}}+\zeta_{2}\right), & \text { if } j \neq k \\ \frac{\eta_{j}}{n}\left(\frac{\zeta_{1}}{\sqrt{1-n a_{0}^{2}}}-(n-1) \zeta_{2}\right), & \text { if } j=k\end{cases}
$$

for some $a_{0} \in \mathbb{R}, a_{0}^{2}<\frac{1}{n}$, and $\zeta_{1}, \zeta_{2}, \eta_{j} \in \mathbb{T}, \eta_{j}^{m}=1, j=1, \ldots, n$. Condition (17) implies that $\varphi$ is of the form (7).

- $N_{Q} \geqslant 2$ and each of the entries in the reduced matrix $\hat{Q}$ appears in some row at least twice. Because of the symmetry of the polynomial (14) we infer that it is the case in every row. Denote all entries of the reduced matrix $\hat{Q}$ by $\alpha_{1}, \ldots, \alpha_{N_{Q}}$ and let $N_{\alpha_{j}}$ denote the number of entries of the matrix $\hat{Q}$ equal to $\alpha_{j}$ in any row. By the symmetry, the polynomial (14) has to have $n ! / \prod_{j=1}^{N_{Q}} N_{\alpha_{j}}$ ! different factors. Since $N_{\alpha_{j}} \geqslant 2, j=1, \ldots, N_{Q}$, we easily conclude that

$$
\frac{n !}{\prod_{j=1}^{N_{Q}} N_{\alpha_{j}} !}>n
$$

- a contradiction. 
Case (i-ii). Suppose now that $N_{j}=n-1 \geqslant 2, j=1,2, \ldots, n$. Then the symmetry of the polynomial (14) implies that for any $k$ there is a unique $j=j(k)$ such that $q_{j, k}=0$. We consider two subcases.

- Assume that $N_{Q}=2$. Thus, if $q_{j, k} \neq 0$ then $q_{j, k}=\eta_{j} \alpha$ for some $\eta_{j} \in$ $\mathbb{T}, \eta_{j}^{m}=1, j=1, \ldots, n$, and $\alpha \in \mathbb{C}_{*}$. Hence we may assume that $q_{j, k}$ 's satisfy condition (15) with $\alpha \neq \beta=0$. Then equalities (11) give (16) with $\beta=0$, which, after elementary calculation, implies that $\alpha \in \mathbb{T}$ and $a_{0}= \pm \sqrt{n-2} /(n-1)$. Note that in this case $\varphi$ is of the form (7) with $\zeta_{1}=\zeta_{2}$ and $a_{0}= \pm \sqrt{n-2} /(n-1)$.

- Assume now that $N_{Q} \geqslant 3$. Then the symmetric polynomial (14) has to have at least $2 n$ different factors - a contradiction.

Case (i-iii). If $N_{j}=1, j=1,2, \ldots, n$, then for any $k$ there is a unique $j=j(k)$ such that $q_{j, k} \neq 0$. Consequently,

$$
\varphi\left(z_{1}, \ldots, z_{n}\right)=\left(q_{1, \sigma(1)}\left(z_{\sigma(1)}-a_{0}\right), \ldots, q_{n, \sigma(n)}\left(z_{\sigma(n)}-a_{0}\right)\right)
$$

for some $\sigma \in \mathfrak{S}_{n}$. First equality in (11) implies that $a_{0}=0$ and $\left|q_{j, \sigma(j)}\right|=$ $1, j=1, \ldots, n$. Repeating the argument from part (a) we conclude that $\varphi$ is of the form (6).

Ad (ii). We repeat the reasoning from the case (i). Since $a=0$, the case $N_{j}=1, j=1, \ldots, n$, implies that $\varphi$ is of the form (6).

Suppose now that $N_{j}=N \in\{n-1, n\}, j=1,2, \ldots, n$. Equality (5) implies that $\pi_{n}\left(\varphi^{m}(z)\right)=\pi_{n}\left(\varphi^{m}(\xi z)\right)$ for any $z \in \mathbb{B}_{n}$ and $\xi=\left(\xi_{1}, \ldots, \xi_{n}\right) \in \mathbb{T}^{n}$, $\xi_{j}^{l}=1, j=1, \ldots, n, l \geqslant 2$.

- If $N=n$, then the polynomial (14) has to have at least $2^{n-1}$ differentup to multiplicative constant-linear factors. Consequently, $2^{n-1} \leqslant n-\mathrm{a}$ contradiction;

- If $N=n-1 \neq 1$, then the polynomial (14) has to have at least $2 n$ different-up to multiplicative constant-linear factors. Consequently, $2 n \leqslant$ $n-$ a contradiction.

Ad (iii). In this case condition (13) means that for any $\sigma \in \mathfrak{S}_{2}$ there are $\tau \in \mathfrak{S}_{2}$ and $\eta=\left(\eta_{1}, \eta_{2}\right) \in \mathbb{T}^{2}, \eta_{j}^{m}=1, j=1,2$, such that

$$
\varphi(z)=\eta \varphi_{\tau}\left(z_{\sigma}\right), \quad z \in \mathbb{B}_{2} .
$$

Without loss of generality we may assume that $\sigma \neq \mathrm{id}$. We consider two cases.

Case $\tau=\mathrm{id}$. Then (18) for $z=\left(0, a_{0}\right)$ and $z=\left(a_{0}, 0\right)$ implies $a_{0}=0$ or

$$
\left\{\begin{array}{l}
q_{1,1}=\eta_{1} q_{1,2} \\
q_{2,1}=\eta_{2} q_{2,2} \\
q_{1,2}=\eta_{1} q_{1,1} \\
q_{2,2}=\eta_{2} q_{2,1}
\end{array} .\right.
$$

If $a_{0}=0$, then (18) for $z=\left(0, z_{2}\right), z_{2} \neq 0$, and $z=\left(z_{1}, 0\right), z_{1} \neq 0$, implies again condition (19). Observe that $\eta_{1}^{2}=\eta_{2}^{2}=1$.

Consequently, we are looking for matrix $Q$ satisfying (11) and (19). Elementary calculation shows that 
- if $m$ is odd, then $\eta_{j}=1, j=1,2$, and (19) leads to contradiction with (11);

- if $m$ is even, then $Q$ satisfies (11) iff $\eta_{1} \eta_{2}=-1$ and $a_{0} \in \mathbb{R}, a_{0}^{2}<\frac{1}{2}$. In this case $Q$ is, up to permutation of the rows, of the form

$$
Q=\frac{1}{\sqrt{2}}\left(\begin{array}{cc}
\frac{\zeta_{1}}{\sqrt{1-2 a_{0}^{2}}} & \frac{\zeta_{1}}{\sqrt{1-2 a_{0}^{2}}} \\
\zeta_{2} & -\zeta_{2}
\end{array}\right), \quad \zeta_{1}, \zeta_{2} \in \mathbb{T} .
$$

Condition (20) implies that $\varphi$ is of the form (8).

Case $\tau \neq \mathrm{id}$. Then (18) for $z=\left(0, a_{0}\right)$ and $z=\left(\left(a_{0}, 0\right)\right)$ implies $a_{0}=0$ or

$$
\left\{\begin{array}{l}
q_{1,1}=\eta_{1} q_{2,2} \\
q_{2,1}=\eta_{2} q_{1,2} \\
q_{1,2}=\eta_{1} q_{2,1} \\
q_{2,2}=\eta_{2} q_{1,1}
\end{array} .\right.
$$

If $a_{0}=0$, then (18) for $z=\left(0, z_{2}\right), z_{2} \neq 0$, and $z=\left(z_{1}, 0\right), z_{1} \neq 0$, implies again condition (21), which is equal to (15). Consequently, we infer that the matrix $Q$ is of the form (17), i.e., $\varphi$ is of the form (7).

Ad (iv). We consider two cases.

Case $\sigma=\mathrm{id}, \xi=(1,-1)$. It follows immediately from (13) that $\tau \neq \mathrm{id}$. Then (13) for $z=\left(z_{1}, 0\right), z_{1} \neq 0$, and $z=\left(0, z_{2}\right), z_{2} \neq 0$, implies

$$
\left\{\begin{array}{l}
q_{1,1}=\eta_{1} q_{1,2} \\
q_{2,1}=\eta_{2} q_{1,1} \\
q_{1,2}=-\eta_{1} q_{2,2} \\
q_{2,2}=-\eta_{2} q_{1,2}
\end{array} .\right.
$$

In particular, $\eta_{1} \eta_{2}=1$. Since $Q$ is unitary, it follows that

$$
Q=\frac{1}{\sqrt{2}}\left(\begin{array}{cc}
\zeta_{1} & \zeta_{2} \\
\eta \zeta_{1} & -\eta \zeta_{2}
\end{array}\right), \quad \zeta_{1}, \zeta_{2}, \eta \in \mathbb{T}, \eta^{m}=1 .
$$

Case $\sigma \neq \mathrm{id}, \xi=(1,-1)$. We consider two subcases.

- $\tau=$ id. Then (13) for $z=\left(z_{1}, 0\right), z_{1} \neq 0$, and $z=\left(0, z_{2}\right), z_{2} \neq 0$, implies

$$
\left\{\begin{array}{l}
q_{1,1}=-\eta_{1} q_{1,2} \\
q_{2,1}=-\eta_{2} q_{2,2} \\
q_{1,2}=\eta_{1} q_{1,1} \\
q_{2,2}=\eta_{2} q_{2,1}
\end{array}\right.
$$

In particular, $\eta_{1}^{2}=\eta_{2}^{2}=-1$, which is possible only iff $4 \mid m$. If it is the case, then $Q$ is unitary iff, up to permutation of rows,

$$
Q=\frac{1}{\sqrt{2}}\left(\begin{array}{cc}
\zeta_{1} & i \zeta_{1} \\
\zeta_{2} & -i \zeta_{2}
\end{array}\right), \quad \zeta_{1}, \zeta_{2} \in \mathbb{T}
$$


- $\tau \neq$ id. Then (13) for $z=\left(z_{1}, 0\right), z_{1} \neq 0$, and $z=\left(0, z_{2}\right), z_{2} \neq 0$, implies

$$
\left\{\begin{array}{l}
q_{1,1}=-\eta_{1} q_{2,2} \\
q_{2,1}=-\eta_{2} q_{1,2} \\
q_{1,2}=\eta_{1} q_{2,1} \\
q_{2,2}=\eta_{2} q_{1,1}
\end{array}\right.
$$

In particular, $\eta_{1} \eta_{2}=-1$, whence $\eta_{1}=-\bar{\eta}_{2}$. then $Q$ is unitary iff, up to permutation of rows,

$$
Q=\zeta\left(\begin{array}{cc}
r & \sqrt{1-r^{2}} \\
-\eta \sqrt{1-r^{2}} & \eta r
\end{array}\right), \quad \zeta, \eta \in \mathbb{T}, \eta^{m}=1, r \in[0,1] .
$$

Straightforward calculation shows that the only unitary matrices satisfying (22) and ((23) or (24)) are, up to permutation of rows, of the form

$$
Q=\frac{\zeta}{\sqrt{2}}\left(\begin{array}{cc}
1 & 1 \\
\eta & -\eta
\end{array}\right), \quad \zeta, \eta \in \mathbb{T}, \eta^{m}=1 .
$$

It is easy to see that unitary automorphism $\varphi$ of $\mathbb{B}_{2}$ represented by matrix (25) satisfies condition (13). Since (13) is also satisfied by the automorphism $\varphi$ of the form (6), the proof of the case (iv) is finished.

Acknowledgements. The author is greatly indebted to E. Kosiński for many stimulating conversations.

Open Access. This article is distributed under the terms of the Creative Commons Attribution Noncommercial License which permits any noncommercial use, distribution, and reproduction in any medium, provided the original author(s) and source are credited.

\section{References}

[1] J. Agler And N. J. Young, The hyperbolic geometry of the symmetrized bidisc, J. Geom. Anal. 14 (2004), 375-403.

[2] H. Alexander, Holomorphic mappings from the ball and polydisc, Math. Ann. 209 (1974), 249-256.

[3] H. Alexander, Proper holomorphic mappings in $\mathbb{C}^{n}$, Indiana Univ. Math. J. 26 (1977), 137-146.

[4] C. Costara, The symmetrized bidisc and Lempert's theorem, Bull. London Math. Soc. 36 (2004), 656-662.

[5] G. Dini and A. Selvaggi Primicerio, Proper holomorphic mappings between generalized pseudoellipsoids, Ann. Mat. Pura Appl. 158 (1991), 219-229.

[6] A. Edigarian and W. Zwonek, Geometry of the symmetrized polydisc, Arch. Math. 84 (2005), 364-374.

[7] Ł. Kosiński, Geometry of quasi-circular domains and applications to tetrablock, Proc. Amer. Math. Soc. 139 (2011), 559-569.

[8] M. LANDUCCI, On the proper holomorphic equivalence for a class of pseudoconvex domains, Trans. Amer. Math. Soc. 282 (1984), 807-811. 
[9] I. G. Macdonald, Symmetric Functions and Hall Polynomials, 2nd ed., Oxford, Clarendon Press, 1995.

[10] N. Nikolov, P. Pflug, And W. Zwonek, An example of a $\mathbb{C}$-convex domain which is not biholomorphic to a convex domain, Math. Scand. 102 (2008), 149-155.

[11] E. M. Stein, Boundary behaviour of holomorphic functions of several complex variables, Math. Notes No. 11, Princeton, 1972.

[12] P. ZapaŁowski, Geometry of symmetrized ellipsoids, Univ. Iagel. Acta Math. 46 (2008), 105-116.

PaWeŁ ZapaŁowsKi

Institute of Mathematics,

Jagiellonian University,

Łojasiewicza 6,

30-348 Kraków, Poland

e-mail: Pawel.Zapalowski@im.uj.edu.pl

Received: 10 March 2011 